\title{
On the Conditional Hardness of Coloring a 4-colorable Graph with Super-Constant Number of Colors
}

\author{
Irit Dinur * $\quad$ Igor Shinkar *
}

\begin{abstract}
For $3 \leq q<Q$ we consider the ApproxColoring $(q, Q)$ problem of deciding for a given graph $G$ whether $\chi(G) \leq q$ or $\chi(G) \geq Q$. It was show in [DMR09] that the problem ApproxColoring $(q, Q)$ is NP-hard for $q=3,4$ and arbitrary large constant $Q$ under variants of the Unique Games Conjecture.

In this paper we give a tighter analysis of the reduction of [DMR09] from Unique Games to the APPROXCOLORING problem. We find that (under appropriate conjecture) a careful calculation of the parameters in [DMR09] implies hardness of coloring a 4colorable graph with $\log ^{c}(\log (n))$ colors for some constant $c>0$. By improving the analysis of the reduction we show hardness of coloring a 4-colorable graph with $\log ^{c}(n)$ colors for some constant $c>0$.

The main technical contribution of the paper is a variant of the Majority is Stablest Theorem, which says that among all balanced functions in which every coordinate has $o(1)$ influence, the Majority function has the largest noise stability. We adapt the theorem for our applications to get a better dependency between the parameters required for the reduction.
\end{abstract}

Keywords: graph coloring, hardness of approximation, majority is stablest

${ }^{*}$ Weizmann Institute of Science. Email: irit.dinur@weizmann.ac.il,igor.shinkar@weizmann.ac.il. Both authors' work supported by ISF grant 1179/09, BSF grant 2008293, and ERC starting grant 239985. 


\section{Introduction}

Graph Coloring is one of the most fundamental problems in combinatorics and computer science. A graph $G$ on $n$ vertices is said to be $q$-colorable if there is an assignment of labels $\{1, \ldots, q\}$ to the vertices of $G$, so that every two neighboring vertices receive different colors. The chromatic number of $G$, denoted by $\chi(G)$, is the minimal number $q$ such that $G$ is $q$-colorable. For $q<Q$ we consider the problem $\operatorname{ApproxColoring}(q, Q)$ : Given a graph $G$, decide whether $\chi(G) \leq q$ or $\chi(G) \geq Q$. It is well known that for any constant $q \geq 3$ the problem ApproxColoring $(q, q+1)$ is NP-hard [Kar72]. If we consider $q$ to be some small fixed number (e.g., 3 or 4 ), there is a huge gap between the values of $Q$ for which an efficient algorithm for the problem is known, and that for which hardness results exist. For example, for $q=3$ the best known polynomial time algorithm is due to Kawarabayashi and Thorup [KT12] who solve the problem for $Q=O\left(n^{0.2049}\right)$ colors (see also [KMS98, BK97, AC06, Chl07]). On the other hand, the strongest known hardness result shows that the problem is NP-hard for $Q=5$ (see [GK04], [KLS00]). That is, for $q=3$ the problem is open for all $5<Q<O\left(n^{0.2072}\right)$.

Many inapproximability results are shown by a reduction from the PCP theorem [AS98, $\mathrm{ALM}^{+} 98$ ], formulated in terms of the Label Cover problem. An instance $\Phi$ of the Label Cover problem is a bipartite graph $(V \cup W, E)$, label sets $R_{V}$ and $R_{W}$, and a constraint set $\Pi=\left\{\pi_{e} \subset R_{V} \times R_{W}: e \in E\right\}$. The goal is to find a labeling that maximizes the fraction of satisfied constraints, i.e., the fraction of constraints that are satisfied by the labels on the relevant vertices. The value of instance $\Phi$, denoted by $\operatorname{val}(\Phi)$, is the fraction of satisfied constraints under such assignment. A Label Cover instance has " $d$-to- 1 property" if the label sets are $R_{V}=\{1, \ldots, R\}, R_{W}=\{1, \ldots, d R\}$ for some $R \in \mathbb{N}$, and the constraints are projections $\pi_{e}: R_{W} \rightarrow R_{V}$, such that for every $a \in R_{V}$ there are $d$ values $b \in R_{W}$ that satisfy $(a, b) \in \pi_{e}$. Khot [Kho02] has made following conjecture.

Conjecture 1.1 ( $d$-to-1 Conjecture [Kho02]) For any $\epsilon>0$ there is $R=R(\epsilon)$ such that given a d-to-1 Label Cover instance $\Phi=(V \cup W, E, \Pi)$ with label sets $R_{V}=\{1, \ldots, R\}$ and $R_{W}=\{1, \ldots, d R\}$, it is $N P$-hard to distinguish between the case where $\operatorname{val}(\Phi)=1$ and the case where $\operatorname{val}(\Phi)<\epsilon$.

\subsection{Our Result}

Assuming Khot's 2-to-1 conjecture it is shown in [DMR09] that the problem of coloring a 4-colorable graph with any constant number of colors is NP-hard. We give a quantitative version of this result. Specifically, we analyze the dependency between the inapproximability factor of the 2-to-1 Label Cover problem and the number $Q$ of colors with which it is still hard to color a 4-colorable graph. Our main result is the following theorem:

Theorem 1.2 Suppose that given a 2-to-1 Label Cover instance $\Phi$ with $n$ vertices and label sets of size $\left|R_{V}\right|,\left|R_{W}\right|=O(\log (n))$, there is no polynomial time algorithm that distinguishes between the case where val $(\Phi)=1$ and the case where $\operatorname{val}(\Phi)<\frac{1}{f(n)}$ for some $f(n)$. Then, there exists no polynomial time algorithm that colors a 4-colorable graph on $N$ vertices with $\left(f\left(N^{c}\right)\right)^{c}$ colors for some constant $c>0$.

For example, if $f(n)=\log ^{\delta}(n)$, then there exists no poly-time algorithm that colors a 4-colorable graph on $N$-vertices with $\log ^{\delta^{\prime}}(N)$ colors for some $\delta^{\prime}>0$.

The theorem improves the dependency between the inapproximability factor of 2-to-1 Label Cover and the hardness of the graph coloring problem. For comparison, the (implicit) 
dependency in [DMR09] is logarithmic, i.e., the soundness of $1 / f(n)$ in the Label Cover is translated into hardness of coloring a 4-colorable graph with $\Omega(\log (f(n)))$ colors.

The main technical contribution of the paper is the following theorem. It follows from a variation of the Majority is Stablest Theorem, which has been developed in the paper of Mossel et al. [MOO10].

Theorem 1.3 Let $q$ be a fixed integer and let $T$ be a symmetric Markov operator on $[q]$ with spectral radius $\rho=\rho(T)<1$. Then, for any $\epsilon>0$ there exist $\delta=\epsilon^{O(1)}$ and $k=O(\log (1 / \epsilon))$, where the constants in the $O()$ notation depend only on $\rho$ and $q$, such that the following holds: For any two functions $f, g:[q]^{n} \rightarrow[0,1]$ if

$$
\mathbb{E}[f]>\epsilon \quad \mathbb{E}[g]>\epsilon \quad \text { and } \quad\left\langle f, T^{\otimes n} g\right\rangle=0,
$$

then

$$
\exists i \in\{1, \ldots, n\} \quad \text { such that } \quad \operatorname{Inf}_{i}^{\leq k}(f) \geq \delta \quad \text { and } \quad \operatorname{Inf}_{i}^{\leq k}(g) \geq \delta .
$$

In the analogous theorem in [DMR09, Corollary 4.12], the (implicit) dependence between $\delta$ and $\epsilon$ is exponential, that is, $\delta=\exp (-1 / \epsilon)$. Our contribution is a new analysis that gives a polynomial dependence between the parameters, which in turn allows us to improve the inapproximability factor in Theorem 1.2 to be polynomial rather that logarithmic in the assumed gap of the 2-to-1 Label Cover problem.

In Section 3 we prove a variant of the Majority is Stablest Theorem with adjustments for our purposes, which allows us to conclude Theorem 1.3. For the sake of completeness, in Section 4 we present the reduction of [DMR09] from 2-to-1 Label Cover problem to the ApPROXCOLORING problem, and work out the parameters required for Theorem 1.2.

\section{Preliminaries}

\subsection{Functions on the $q$-ary hypercube}

Let $q$ be a fixed integer. Let $[q]$ denote the set $\{0, \ldots, q-1\}$. For an element $x \in[q]^{n}$ denote by $|x|$ the number of nonzero coordinates of $x$. Consider the space of real valued function with domain $[q]$, or, equivalently, a vector space $\mathbb{R}^{q}$ with inner product defined as

$$
\langle v, w\rangle=\mathbb{E}[v w]=\frac{1}{q} \sum_{i=1}^{q} v_{i} w_{i}
$$

and norm of a vector defined as

$$
\|v\|=\sqrt{\langle v, v\rangle} .
$$

Let $\alpha_{0}=\mathbf{1}, \alpha_{1}, \ldots, \alpha_{q-1}$ be some orthonormal basis of $\mathbb{R}^{q}$. It defines naturally an orthonormal basis of $\mathbb{R}^{q^{n}}$ by applying the $n$-fold tensor product. It is easy to see that the set $\left\{\alpha_{x}=\alpha_{x_{1}} \otimes \alpha_{x_{2}} \otimes \cdots \otimes \alpha_{x_{n}} \in \mathbb{R}^{q^{n}}: x \in[q]^{n}\right\}$ is indeed an orthonormal basis of $\mathbb{R}^{q^{n}}$. Equivalently, we may think of $\alpha_{x}$ as a function $\alpha_{x}:[q]^{n} \rightarrow \mathbb{R}$ defined by $\alpha_{x}(y)=\prod_{i=1}^{n} \alpha_{x_{i}}\left(y_{i}\right)$. Thus, any function $f:[q]^{n} \rightarrow \mathbb{R}$ can be written as

$$
f=\sum_{x \in[q]^{n}} \hat{f}\left(\alpha_{x}\right) \alpha_{x}
$$

Next we define the notion of influence of a variable on a function, introduced to computer science by Ben-Or and Linial in [BOL89]. 
Definition 2.1 Let $f:[q]^{n} \rightarrow \mathbb{R}$ be a function on a q-ary hypercube. The influence of the $x_{i}$ on $f$, is defined as

$$
\operatorname{Inf}_{i}(f)=\mathbb{E}_{x \backslash i}\left[\operatorname{Var}_{x_{i}}\left[f(x) \mid x_{1}, \ldots, x_{i-1}, x_{i+1}, \ldots, x_{n}\right]\right],
$$

where $x_{1}, \ldots, x_{n}$ are uniformly distributed in $[q]$.

Some standard formulas are easily checkable using independence and orthonormality.

Proposition 2.2 Let $f:[q]^{n} \rightarrow \mathbb{R}$ be as in Eq. (1). Then

$$
\begin{gathered}
\mathbb{E}[f]=\hat{f}\left(\alpha_{0}\right), \quad \mathbb{E}\left[f^{2}\right]=\sum_{x} \hat{f}\left(\alpha_{x}\right)^{2}, \\
\operatorname{Var}[f]=\sum_{|x|>0} \hat{f}\left(\alpha_{x}\right)^{2}, \quad \operatorname{Inf}_{i}(f)=\sum_{x: x_{i} \neq 0} \hat{f}\left(\alpha_{x}\right)^{2} .
\end{gathered}
$$

Analogously, we can define "low-degree influence". This notion is useful in PCPs due to the fact that a bounded function cannot have too many coordinates with non-negligible low-degree influences.

Definition 2.3 Let $f:[q]^{n} \rightarrow \mathbb{R}$ be a function. Define the d-low-degree influence of $f$ as

$$
\operatorname{Inf}_{i}^{\leq d}(f)=\sum_{x: x_{i} \neq 0,|x| \leq d} \hat{f}\left(\alpha_{x}\right)^{2} .
$$

The remark above follows from the following easy proposition.

Proposition 2.4 Let $f:[q]^{n} \rightarrow \mathbb{R}$ be as in Eq. (1). Then

$$
\sum_{i} \operatorname{Inf}_{i}^{\leq d}(f) \leq d \cdot \operatorname{Var}[f]
$$

In particular for $f:[q]^{n} \rightarrow[-1,1]$ it holds that

$$
\sum_{i} \operatorname{Inf}_{i}^{\leq d}(f) \leq d
$$

and thus there are at most $d / \epsilon$ variables $x_{i}$ with $\operatorname{Inf}_{i}^{\leq d}(f) \geq \epsilon$.

Instead of picking $x$ at random, changing one coordinate, and seeing how it changes the value of $f$, we can change a constant fraction (in expectation) of the coordinates.

Definition 2.5 Let $f:[q]^{n} \rightarrow \mathbb{R}$, and let $\rho \in[0,1]$. Let $x \in[q]^{n}$ be chosen uniformly at random, and choose each coordinate $y_{i}$ is independently to be $x_{i}$ with probability $\rho$ and uniformly random element of $[q]$ otherwise. Define the noise stability of $f$ to be

$$
\mathbb{S}_{\rho}(f)=\mathbb{E}[f(x) f(y)] .
$$

Analogously, we generalize the notion of stability with respect to two functions:

$$
\mathbb{S}_{\rho}(f, g)=\mathbb{E}[f(x) g(y)]
$$


The notion above can be also considered as following: For any $\rho \in[0,1]$ define the following Markov operator on $[q]$ (called the Bonami-Beckner operator).

$$
T_{\rho}=\left(\begin{array}{cccc}
\rho+\frac{1-\rho}{q} & \frac{1-\rho}{q} & \ldots & \frac{1-\rho}{q} \\
\vdots & \ddots & & \\
\vdots & & \ddots & \\
\frac{1-\rho}{q} & \ldots & \frac{1-\rho}{q} & \rho+\frac{1-\rho}{q}
\end{array}\right) .
$$

We note that $T_{\rho} \mathbf{1}=\mathbf{1}$ and $T_{\rho} v=\rho \cdot v$ for any vector $v \perp \mathbf{1}$. In particular holds $T_{1}(f)=f$ and $T_{0}(f)=\mathbb{E}[f]$. The following formulas are standard and easily checkable.

Proposition 2.6 Let $f, g:[q]^{n} \rightarrow \mathbb{R}$ be as in Eq. (1) with respect to some orthonormal basis $\left\{\alpha_{i}\right\}$. Then

$$
T_{\rho}^{\otimes n}(f)=\sum_{x} \rho^{|x|} \hat{f}\left(\alpha_{x}\right) \alpha_{x},
$$

and by orthonormality

$$
\mathbb{S}_{\rho}(f, g)=\left\langle f, T_{\rho}^{\otimes n} g\right\rangle=\sum_{x} \rho^{|x|} \hat{f}\left(\alpha_{x}\right) \hat{g}\left(\alpha_{x}\right) .
$$

By applying $T_{\rho}$ on a function $f:[q]^{n} \rightarrow[0,1]$, the weight of $f$ on higher levels reduces exponentially. More precisely if $g=T_{\rho} f$, then $\sum_{x:|x| \geq k} \hat{g}\left(\alpha_{x}\right)^{2} \leq \rho^{2 k} \sum_{x} \hat{f}\left(\alpha_{x}\right)^{2} \leq \rho^{2 k}$. We think of $T_{\rho}$ as a smoothing operator.

Definition 2.7 Let $g:[q]^{n} \rightarrow \mathbb{R}$, and let $\eta \in(0,1)$. We say that $g$ is $\eta$-smooth if $\sum_{x:|x| \geq k} \hat{g}\left(\alpha_{x}\right)^{2} \leq \eta^{k}$ for all $k \geq 0$.

\subsection{Functions in Gaussian space}

Before we continue, we need to define some basic notions in $L^{2}\left(\mathbb{R}^{n}, \gamma\right)$, the space of real valued functions with domain $\mathbb{R}^{n}$ equipped with the standard Gaussian measure. The density function of the standard normal distribution is $\gamma(x)=\frac{1}{(2 \pi)^{n / 2}} \exp \left(-\frac{\|x\|^{2}}{2}\right)$, and the inner product is defined as

$$
\langle f, g\rangle=\mathbb{E}_{\gamma}[f g]=\int_{R^{n}} f(x) g(x) \gamma(x) d x .
$$

For $\rho \in[-1,1]$ denote by $U_{\rho}$ the Ornstein-Uhlenbeck operator

$$
\left(U_{\rho} f\right)(x)=\mathbb{E}_{y \sim \gamma}\left[f\left(\rho x+\sqrt{1-\rho^{2}} y\right)\right] .
$$

For $\mu \in(0,1)$ define an indicator of half space function $L^{2}(\mathbb{R}, \gamma)$ as

$$
F_{\mu}(x)=\mathbf{1}_{x<\Phi^{-1}(\mu)}(x),
$$

where $\Phi(t)=\int_{-\infty}^{t} \gamma(x) d x$ is the cumulative distribution function.

A useful quantity that will appear later is $\left\langle F_{\epsilon}, U_{\rho}\left(1-F_{1-\epsilon}\right)\right\rangle$, where $\rho \in(0,1)$. Observe that $\left\langle F_{\epsilon}, U_{\rho}\left(1-F_{1-\epsilon}\right)\right\rangle=\left\langle F_{\epsilon}, U_{-\rho} F_{\epsilon}\right\rangle=\operatorname{Pr}\left[X<\Phi^{-1}(\epsilon), Y<\Phi^{-1}(\epsilon)\right]$, where $X$ and $Y$ are $(-\rho)$-correlated normal random variables with mean 0 and variance 1 . That is, we have $X \sim N(0,1)$, and for independent random variable $Z \sim N(0,1)$ the random variable $Y$ is defined to be $-\rho X+\sqrt{1-\rho^{2}} Z$. 
It can be found in the literature (see, e.g., in [RR01], [KPW04]) that as $\epsilon \rightarrow 0$ we have

$$
\left\langle F_{\epsilon}, U_{-\rho} F_{\epsilon}\right\rangle \sim \epsilon^{2 /(1-\rho)}(4 \pi \ln (1 / \epsilon))^{\rho /(1-\rho)} \frac{(1-\rho)^{3 / 2}}{(1+\rho)^{1 / 2}} .
$$

In particular if $\rho$ is some constant bounded below 1 , then

$$
\left\langle F_{\epsilon}, U_{\rho}\left(1-F_{1-\epsilon}\right)\right\rangle=\operatorname{poly}(\epsilon) .
$$

\subsection{The Majority is Stablest Theorem}

The Majority is Stablest Theorem [MOO10] roughly says that for all functions $f:[q]^{n} \rightarrow$ $[0,1]$ in which every coordinate has $o(1)$ influence, the noise stability of $f$ is bounded by some function of $\mathbb{E}[f]$. More specifically we have the following theorem.

Theorem 2.8 ([MOO10, Theorem 4.4]) Fix $q \geq 2$ and $\rho \in[0,1]$. Then, for any $\epsilon>0$ there is a small enough $\delta=\delta(\epsilon, \rho, q)$ such that for any function $f:[q]^{n} \rightarrow[0,1]$ that satisfies

$$
\operatorname{Inf}_{i}(f) \leq \delta \quad \forall i \in\{1, \ldots, n\}
$$

it holds that

$$
\mathbb{S}_{\rho}(f) \leq\left\langle F_{\mathbb{E}[f]}, U_{\rho} F_{\mathbb{E}[f]}\right\rangle+\epsilon .
$$

In particular case of $q=2$ and balanced functions $f:\{0,1\} \rightarrow\{0,1\}$ the theorem states that if

$$
\mathbb{S}_{\rho}(f)>\left\langle F_{\mathbb{E}[f]}, U_{\rho} F_{\mathbb{E}[f]}\right\rangle+\epsilon=\frac{1}{4}+\frac{1}{2 \pi} \arcsin \rho+\epsilon=\mathbb{S}_{\rho}(M a j)+\epsilon
$$

then $f$ has some coordinate with non-negligible influence. That is among all balanced boolean functions in which every coordinate has o(1) influence, the Majority function has the largest noise stability.

This theorem is generalized in [DMR09] in two directions: the stability is defined with respect to two functions, and for any Markov operator $T$ on $[q]$ (not only for $T_{\rho}$ ). The idea is that given a symmetric Markov operator $T$ with eigenvalues $1=\lambda_{0}>\lambda_{1} \geq \cdots \geq \lambda_{q-1}$, it is enough to bound its spectral radius $\rho=\rho(T)=\max \left\{\left|\lambda_{1}\right|,\left|\lambda_{q-1}\right|\right\}$ below 1. Suppose we are given a symmetric Markov operator $T$ on $[q]$ with spectral radius $\rho<1$, and two functions $f, g:[q]^{n} \rightarrow[0,1]$ that satisfy the inequality

$$
\left\langle f, T^{\otimes n} g\right\rangle>\left\langle F_{\mathbb{E}[f]}, U_{\rho} F_{\mathbb{E}[g]}\right\rangle+\epsilon .
$$

The main technical result in [DMR09, Theorem 3.1] says that in such case $f$ and $g$ have a common coordinate with non-negligible influence. In our setup, however, we consider functions $f$ and $g$ with small expectation and $\rho$ some fixed constant. This allows us to assume $\left\langle f, T^{\otimes n} g\right\rangle>\left\langle F_{\mathbb{E}[f]}, U_{\rho^{\prime}} F_{\mathbb{E}[g]}\right\rangle+\epsilon$ for some $\rho^{\prime}>\rho$, and conclude that $f$ and $g$ have a common coordinate with relatively large influence on both functions. The exact formulation and the proof appear in the next section.

\section{A Variant of the Majority is Stablest Theorem}

In this section we prove our main technical result stated in Theorem 3.1, which is used in order to prove Theorem 1.3. This is a variant of [DMR09, Theorem 3.1] that we adjust for our purposes. 
Let $q$ be a fixed integer, and let $T$ be a symmetric Markov operator on $[q]$ with eigenvalues $1=\lambda_{0}>\lambda_{1} \geq \cdots \geq \lambda_{q-1}>-1$, and let $\alpha_{0}=1, \alpha_{1}, \ldots, \alpha_{q-1}$ be the corresponding eigenvectors. Denote the spectral radius of $T$ by $\rho=\rho(T)=\max \left\{\left|\lambda_{1}\right|,\left|\lambda_{q-1}\right|\right\}<1$.

Now suppose we are given two functions $f, g:[q]^{n} \rightarrow[0,1]$ that do not have a common influential coordinate. We show that it implies bounds on the quantity $\left\langle f, T^{\otimes n} g\right\rangle$.

Theorem 3.1 Let $q$ be a fixed integer, and let $T$ be a symmetric Markov operator on $[q]$ such that $\rho=\rho(T)<1$ and let $\rho^{\prime} \in(\rho, 1)$. Then, for any $\epsilon>0$ there are $\delta=\epsilon^{O(1)}$ and $k=O(\log (1 / \epsilon))$, where the constants in the $O()$ notation depend only on $\frac{\rho}{\rho^{\prime}}$ and $q$, such that the following holds: If $f, g:[q]^{n} \rightarrow[0,1]$ are two functions with $\mu=\mathbb{E}[f]$ and $\nu=\mathbb{E}[g]$ satisfying

$$
\forall i \quad \min \left(\operatorname{Inf}_{i}^{\leq k}(f), \operatorname{Inf}_{i}^{\leq k}(g)\right)<\delta,
$$

then

$$
\left\langle f, T^{\otimes n} g\right\rangle \geq\left\langle F_{\mu}, U_{\rho^{\prime}}\left(1-F_{1-\nu}\right)\right\rangle_{\gamma}-\epsilon
$$

and

$$
\left\langle f, T^{\otimes n} g\right\rangle \leq\left\langle F_{\mu}, U_{\rho^{\prime}} F_{\nu}\right\rangle_{\gamma}+\epsilon .
$$

Observe that compared to the analogous theorem of [DMR09, Theorem 3.1], we gain a better tradeoff between $\epsilon$ and $\delta$. Specifically, we allow $\delta$ to be poly $(\epsilon)$, i.e., not too small, (instead of $\delta=\exp (-1 / \epsilon)$ implicitly appearing in [DMR09]). On the other hand, we get a bound on $\left\langle f, T^{\otimes n} g\right\rangle$ as a function of $\rho^{\prime} \in(\rho, 1)$ rather than $\rho$.

For our application of the theorem, we think of $\rho$ and $\rho^{\prime}$ as constants smaller than 1 , and of $\mu$ and $\nu$ as small quantities compared to $\epsilon$. In this setup, the polynomial dependency between $\epsilon$ and $\delta$ improves the dependency of [MOO10] and [DMR09]. The following corollary proves Theorem 1.3.

Corollary 3.2 Let $q$ be a fixed integer and $T$ be a symmetric Markov operator on $[q]$ with spectral radius $\rho=\rho(T)<1$. Then, for any $\epsilon>0$ there exist $\delta=\epsilon^{O(1)}$ and $k=O(\log (1 / \epsilon))$, where the constants in the $O()$ notation depend only on $\rho$ and $q$, such that the following holds: For any two functions $f, g:[q]^{n} \rightarrow[0,1]$ if

$$
\mathbb{E}[f]>\epsilon \quad \mathbb{E}[g]>\epsilon \quad \text { and } \quad\left\langle f, T^{\otimes n} g\right\rangle=0,
$$

then

$$
\exists i \in\{1, \ldots, n\} \quad \text { such that } \quad \operatorname{Inf}_{i}^{\leq k}(f) \geq \delta \quad \text { and } \quad \operatorname{Inf}_{i}^{\leq k}(g) \geq \delta .
$$

Proof Let $\rho^{\prime}=\sqrt{\rho}$ (note that $\rho<\rho^{\prime}<1$ ), and let $\epsilon_{0}=\left\langle F_{\epsilon}, U_{\rho^{\prime}}\left(1-F_{1-\epsilon}\right)\right\rangle_{\gamma}$. Then

$$
\left\langle f, T^{\otimes n} g\right\rangle=0<\left\langle F_{\mathbb{E}[f]}, U_{\rho^{\prime}}\left(1-F_{1-\mathbb{E}[g]}\right)\right\rangle_{\gamma}-\epsilon_{0} .
$$

By applying the contrapositive of Theorem 3.1 we get $\delta=\operatorname{poly}\left(\epsilon_{0}\right), k=O\left(\log \left(1 / \epsilon_{0}\right)\right)$, and some $i \in\{1, \ldots, n\}$ such that $\operatorname{Inf}_{i}^{\leq k}(f)>\delta$ and $\operatorname{Inf}_{i}^{\leq k}(g)>\delta$. Using Eq. (2) we have $\epsilon_{0}=\left\langle F_{\epsilon}, U_{\rho^{\prime}}\left(1-F_{1-\epsilon}\right)\right\rangle_{\gamma}=\operatorname{poly}(\epsilon)$, where the degree of the polynomial depends only on $\rho^{\prime}$. Therefore, we have $\delta=\operatorname{poly}(\epsilon), k=O(\log (1 / \epsilon))$, as required. 


\subsection{Proof of Theorem 3.1}

Note that Eq. (3) follows from Eq. (4). Indeed, apply Eq. (4) to $1-g$ to obtain

$$
\left\langle f, T^{\otimes n}(1-g)\right\rangle \leq\left\langle F_{\mu}, U_{\rho^{\prime}} F_{1-\nu}\right\rangle_{\gamma}+\epsilon,
$$

and then use the equalities

$$
\left\langle f, T^{\otimes n}(1-g)\right\rangle=\langle f, 1\rangle-\left\langle f, T^{\otimes n} g\right\rangle=\mu-\left\langle f, T^{\otimes n} g\right\rangle=\left\langle F_{\mu}, U_{\rho^{\prime}} 1\right\rangle-\left\langle f, T^{\otimes n} g\right\rangle .
$$

So our goal in this section is to prove Eq. (4).

The following lemma is the first step in the proof of Theorem 3.1. It is proven in Lemma 3.9 in [DMR09], and essentially follows from the one function analogue proven in Theorem 4.4 in [MOO10].

Lemma 3.3 ([DMR09, Lemma 3.9]) Let $T$ be a symmetric Markov operator on $[q]$. Denote its eigenvalues by $1=\lambda_{0}>\lambda_{1} \geq \cdots \geq \lambda_{q-1}>-1$, and let $\alpha_{0}=\mathbf{1}, \alpha_{1}, \ldots, \alpha_{q-1}$ be the corresponding orthonormal eigenbasis. Suppose that the spectral radius of $T$ is $\rho=\rho(T)<1$. Then, for any $\eta<1, \epsilon>0$ there is $\delta=\epsilon^{O\left(\frac{\log (q)}{1-\eta}\right)}$ such that the following holds: Let $f, g:[q]^{n} \rightarrow[0,1]$ be two functions with $\mathbb{E}[f]=\mu, \mathbb{E}[g]=\nu$ with decompositions as in (1). If both functions are $\eta$-smooth, i.e.,

$$
\forall k \sum_{x:|x| \geq k} \hat{f}\left(\alpha_{x}\right)^{2} \leq \eta^{k} \quad \text { and } \quad \forall k \sum_{x:|x| \geq k} \hat{g}\left(\alpha_{x}\right)^{2} \leq \eta^{k},
$$

and all influences in both of them are upper bounded by $\delta$, that is,

$$
\forall i \operatorname{Inf}_{i}(f)<\delta \quad \text { and } \quad \forall i \operatorname{Inf}_{i}(g)<\delta,
$$

then

$$
\left\langle f, T^{\otimes n} g\right\rangle \leq\left\langle F_{\mu}, U_{\rho} F_{\nu}\right\rangle+\epsilon
$$

We next prove Theorem 3.1 by showing that the hypothesis of the theorem implies Eq. (4). In order to prove Theorem 3.1 let $f$ and $g$ be two functions as in the hypothesis of the theorem. Note that we cannot apply Lemma 3.3 directly, as we don't know that all variables of both $f$ and $g$ have small influence and both functions are smooth. We modify the functions so that they satisfy the conditions of Lemma 3.3, while making sure that the quantity $\left\langle f, T^{\otimes n} g\right\rangle$ does not change too much.

In order to satisfy the condition on influences we observe that coordinates that have large influence either on $f$ or on $g$, make small contribution to $\left\langle f, T^{\otimes n} g\right\rangle$. This has been done in [DMR09].

One possible approach to satisfy the smoothness condition is to smooth $f$ and $g$ a little, that is to define $f_{1}=T_{1-\epsilon}(f)$ and $g_{1}=T_{1-\epsilon}(g)$ and show that $\left|\left\langle f, T^{\otimes n} g\right\rangle-\left\langle f_{1}, T^{\otimes n} g_{1}\right\rangle\right|<\epsilon$. But then $f_{1}$ and $g_{1}$ are only $(1-\epsilon)$-smooth, and $\delta$ that we get from Lemma 3.3 will be exponential in $\epsilon .^{1}$

A different approach is to use the fact that $\rho$ is some constant smaller than 1 and to define $f_{1}=T_{\eta}(f)$ and $g_{1}=T_{\eta}(g)$ for some constant $\eta \in(\rho, 1)$. Then $f_{1}$ and $g_{1}$ are $\eta$-smooth

\footnotetext{
${ }^{1}$ Recall that for any $\gamma>0$ the linear operator $T_{\gamma}$ on $\mathbb{R}^{q}$ is defined by: $T_{\gamma} \mathbf{1}=\mathbf{1}$ and $T_{\gamma} v=\gamma v$ for $v \perp \mathbf{1}$. It is easy to see that the operator $S=T T_{\gamma}$ has the same eigenvectors as $T$ and the corresponding eigenvalues are $1=\lambda_{0}>\lambda_{1} \gamma \geq \cdots \geq \lambda_{q-1} \gamma>-1$ (as long as $\gamma<1 / \rho$ ).
} 
functions and $\left\langle f, T^{\otimes n} g\right\rangle=\left\langle f_{1}, S^{\otimes n} g_{1}\right\rangle$ for some operator $S$ whose spectral radius is larger than $\rho(T)$, but still constant smaller than 1. By applying Lemma 3.3 on $f_{1}$ and $g_{1}$ with the operator $S$ we will get $\delta=\operatorname{poly}(\epsilon)$. We now turn to the actual proof of Theorem 3.1.

Proof Set $\eta=\frac{\rho}{\rho^{\prime}}<1$ and denote $S=T T_{\frac{1}{\eta}}$. Then $S$ has the same eigenvectors as $T$, largest eigenvalue 1 and $r(S)=\frac{\rho}{\eta}=\rho^{\prime}<1$. Denote

$$
f_{1}=T_{\sqrt{\eta}} f=\sum \hat{f}\left(\alpha_{x}\right) \eta^{\frac{|x|}{2}} \alpha_{x} \quad \text { and } \quad g_{1}=T_{\sqrt{\eta}} g=\sum \hat{g}\left(\alpha_{x}\right) \eta^{\frac{|x|}{2}} \alpha_{x} .
$$

Using this notation it is easy to see that we can express $\left\langle f, T^{\otimes n} g\right\rangle$ as

$$
\left\langle f, T^{\otimes n} g\right\rangle=\sum_{x} \hat{f}\left(\alpha_{x}\right) \lambda_{x} \hat{g}\left(\alpha_{x}\right)=\left\langle f_{1}, S^{\otimes n} g_{1}\right\rangle .
$$

By applying Lemma 3.3 with operator $S$ and parameters $\eta$ and $\epsilon / 2$, we obtain $\delta^{\prime}=$ $\delta_{3.3}\left(S, \eta, \frac{\epsilon}{2}\right) / 2=\epsilon^{O\left(\frac{1}{1-\eta}\right)}=$ poly $(\epsilon)$, where the degree of the polynomial depends only on $\eta$ and $q$. Let $k=O(\log (1 / \epsilon))$ be such that $\eta^{k}<\min \left(\delta^{\prime}, \epsilon / 4\right)$, and let $\delta=\left(\frac{\epsilon \delta^{\prime}}{8 k}\right)^{2}=\operatorname{poly}(\epsilon)$. We show that these $\delta$ and $k$ satisfy the requirements of Theorem 3.1.

Take two functions $f, g:[q]^{n} \rightarrow[0,1]$ such that $\forall i \min \left(\operatorname{Inf}_{i}^{\leq k}(f), \operatorname{Inf}_{i}^{\leq k}(g)\right)<\delta$. Then $f_{1}$ and $g_{1}$ are $\eta$-smooth and satisfy the same assumption. However, we cannot apply Lemma 3.3 on them with the operator $S$, as the requirement is that influences of all variables in both functions are small. In order overcome this obstacle we define two functions $f_{2}$ and $g_{2}$ with small influences such that the quantities $\left\langle f_{1}, S^{\otimes n} g_{1}\right\rangle$ and $\left\langle f_{2}, S^{\otimes n} g_{2}\right\rangle$ are close to each other. Specifically, let

$$
B_{f}=\left\{i: \operatorname{Inf}_{i}^{\leq k}(f) \geq \delta^{\prime}\right\} \quad B_{g}=\left\{i: \operatorname{Inf}_{i}^{\leq k}(g) \geq \delta^{\prime}\right\} .
$$

Note that by Proposition 2.4 we have $\left|B_{f}\right|,\left|B_{g}\right| \leq k / \delta^{\prime}$. Moreover, $B_{f} \cap B_{g}=\emptyset$ since by the hypothesis holds $\forall i \min \left(\operatorname{Inf}_{i}^{\leq k}(f), \operatorname{Inf}_{i}^{\leq k}(g)\right)<\delta<\delta^{\prime}$. We define the functions $f_{2}, g_{2}:[q]^{n} \rightarrow[0,1]$ to be the average over the coordinates in $B_{f}$ and $B_{g}$ respectively, namely

$$
f_{2}(y)=\underset{y_{i}: i \in B_{f}}{\mathbb{E}}\left[f_{1}(y)\right]=\sum_{x: x_{B_{f}}=0} \hat{f}\left(\alpha_{x}\right) \eta^{\frac{|x|}{2}} \alpha_{x}(y)
$$

and

$$
g_{2}(y)=\underset{y_{i}: i \in B_{g}}{\mathbb{E}}\left[g_{1}(y)\right]=\sum_{x: x_{B_{g}}=0} \hat{g}\left(\alpha_{x}\right) \eta^{\frac{|x|}{2}} \alpha_{x}(y) .
$$

Clearly $\mathbb{E}\left[f_{2}\right]=\mathbb{E}[f]=\mu, \mathbb{E}\left[g_{2}\right]=\mathbb{E}[g]=\nu$. We have $\operatorname{Inf}_{i}\left(f_{2}\right)=0$ for $i \in B_{f}$ and $\operatorname{Inf}_{i}\left(f_{2}\right) \leq \operatorname{Inf}_{i}\left(f_{1}\right) \leq \operatorname{Inf}_{i}^{\leq k}(f)+\eta^{k}<2 \delta^{\prime}$ for $i \notin B_{f}$. Same holds for $g_{2}$. Their smoothness follows from smoothness of $f_{1}, g_{1}$, and we can apply Lemma 3.3 with the operator $S$ to get

$$
\left\langle f_{2}, S^{\otimes n} g_{2}\right\rangle \leq\left\langle F_{\mu}, U_{\rho^{\prime}} F_{\nu}\right\rangle+\epsilon / 2 .
$$

In order to complete the proof it is left to show that

$$
\left|\left\langle f_{1}, S^{\otimes n} g_{1}\right\rangle-\left\langle f_{2}, S^{\otimes n} g_{2}\right\rangle\right| \leq \epsilon / 2 .
$$


Here we use the assumption that no coordinate has significant influence on both functions.

$$
\begin{aligned}
& \left|\left\langle f_{1}, S^{\otimes n} g_{1}\right\rangle-\left\langle f_{2}, S^{\otimes n} g_{2}\right\rangle\right|=\left|\sum_{x: x_{B_{f} \cup B_{g}} \neq 0} \hat{f}\left(\alpha_{x}\right) \hat{g}\left(\alpha_{x}\right)\left(\prod_{i: x_{i} \neq 0} \lambda_{x_{i}}\right)\right| \\
& \leq \sum_{\substack{x:|x| \leq k \\
x: x_{B_{f} \cup B_{g}} \neq 0}}\left|\hat{f}\left(\alpha_{x}\right) \hat{g}\left(\alpha_{x}\right)\right|+\sum_{x:|x|>k}\left|\rho^{|x|} \hat{f}\left(\alpha_{x}\right) \hat{g}\left(\alpha_{x}\right)\right| \\
& {[\rho<\eta] \leq \sum_{i \in B_{f} \cup B_{g}} \sum_{\substack{x:|x| \leq k \\
x_{i} \neq 0}}\left|\hat{f}\left(\alpha_{x}\right) \hat{g}\left(\alpha_{x}\right)\right|+\eta^{k}} \\
& \text { [Cauchy Schwartz] } \leq \sum_{i \in B_{f} \cup B_{g}} \sqrt{\operatorname{Inf}_{i}^{\leq k}(f)} \sqrt{\operatorname{Inf}_{i}^{\leq k}(g)}+\eta^{k} \\
& {\left[i \in B_{f} \Rightarrow \operatorname{Inf}_{i}(g)<\delta\right] \leq\left(\left|B_{f}\right|+\left|B_{g}\right|\right) \sqrt{\delta}+\eta^{k}} \\
& {\left[\left|B_{f}\right|,\left|B_{g}\right| \leq k / \delta^{\prime}, \eta^{k} \leq \epsilon / 4\right] \leq \frac{2 k}{\delta^{\prime}} \frac{\epsilon \delta^{\prime}}{8 k}+\epsilon / 4} \\
& =\epsilon / 2 \text {. }
\end{aligned}
$$

Combining Eq. (5), Eq. (6) and Eq. (7) we get the required result $\left\langle f, T^{\otimes n} g\right\rangle \leq\left\langle F_{\mu}, U_{\rho^{\prime}} F_{\nu}\right\rangle+\epsilon$. This completes the proof of Theorem 3.1.

\section{Proof of Theorem 1.2}

The proof of Theorem 1.2 follows by applying the reduction of [DMR09], and using Theorem 1.3 in order to calculate the exact parameters of the reduction. Specifically we prove the following dependence in the parameters.

Theorem 4.1 There is a reduction from 2-to-1 Label Cover problem to ApproxColoring problem with the following properties: Given an instance of 2-to-1 Label Cover $\Phi=(V \cup$ $W, E, \Pi)$ with label sets of size $R$ and $2 R$, it produces a graph $G^{\prime}$ on $|W| \cdot 4^{2 R}$ vertices.

- If $\operatorname{val}(\Phi)=1$, then $G^{\prime}$ is 4-colorable.

- If $G^{\prime}$ contains an independent set of size $\epsilon$, then $\operatorname{val}(\Phi) \geq \Omega\left(\frac{\epsilon \delta^{2}}{k^{2}}\right)=\operatorname{poly}(\epsilon)$, where $\delta=\epsilon^{O(1)}$ and $k=O(\log (1 / \epsilon))$ as in Theorem 1.3. In other words, if $\operatorname{val}(\Phi) \leq \frac{1}{f(n)}$, then $\chi(G) \geq f^{c}(n)$ for some constant $c>0$.

The running time of the reduction is linear in the size of the output.

Proof The reduction will use the following Markov operator $T$ with domain $\{0,1,2,3\}^{2}$.

Definition 4.2 We define a symmetric Markov operator $T$ with domain $\{0,1,2,3\}^{2}$ such that $T\left(\left(x_{1}, x_{2}\right) \leftrightarrow\left(y_{1}, y_{2}\right)\right)>0$ if and only if $\left\{x_{1}, x_{2}\right\} \cap\left\{y_{1}, y_{2}\right\}=\emptyset$.

The operator has three types of transitions, with transitions probabilities $\beta_{1}, \beta_{2}$, and $\beta_{3}$.

- With probability $\beta_{1}$ we have $(x, x) \leftrightarrow(y, y)$ where $x \neq y$.

- With probability $\beta_{2}$ we have $(x, x) \leftrightarrow(y, z)$ where $x, y, z$ are all different. 
- With probability $\beta_{3}$ we have $(x, y) \leftrightarrow(z, w)$ where $x, y, z, w$ are all different.

For $T$ to be a symmetric Markov operator, we need that $\beta_{1}, \beta_{2}$ and $\beta_{3}$ are non-negative and

$$
3 \beta_{1}+6 \beta_{2}=1, \quad 2 \beta_{2}+2 \beta_{3}=1 .
$$

For example for $\beta_{1}=\frac{1}{12}, \beta_{2}=\frac{1}{8}$, and $\beta_{3}=\frac{3}{8}$ we have $\rho=\rho(T)=5 / 6$

The reduction: We start with a 2-to- 1 Label Cover instance $\Phi=(V \cup W, E, \Pi)$. In addition, as a small technicality, we shall assume that all vertices of $W$ have the same degree. Each $(v, w) \in E$ is associated with a constraint $\pi_{v w}$ such that for each $b \in\{1, \ldots, 2 R\}$ there is a unique $a \in\{1, \ldots, R\}$ such that $(a, b) \in \pi_{v w}$ (we denote $a=\pi_{v w}(b)$ ) and for each $a \in\{1, \ldots, R\}$ there are exactly two $b_{1}, b_{2} \in\{1, \ldots, 2 R\}$ such that $\left(a, b_{i}\right) \in \pi_{v w}$ (denote $\left.\left(b_{1}, b_{2}\right)=\pi_{v w}^{-1}(a)\right)$. We construct $G^{\prime}=\left(V^{\prime}, E^{\prime}\right)$ as follows:

- Each vertex $w \in W$ is replaced by a copy of $\{0,1,2,3\}^{2 R}$ (denoted by $[w]$ ). The set of vertices in $G^{\prime}$ is $V^{\prime}=\bigcup_{w \in W}[w]=W \times\{0,1,2,3\}^{2 R}$.

- Let $T$ be as in Definition 4.2. For every $w_{1}, w_{2} \in W$ that have a common neighbor $v \in V$ let $\pi_{1}, \pi_{2}$ be the corresponding constraints. For $x, y \in\{0,1,2,3\}^{2 R}$ set an edge between $\left(w_{1}, x\right)$ and $\left(w_{2}, y\right)$ if and only if for all $k \in\{1, \ldots, R\}$ it holds that $\left\{x_{i_{1}}, x_{j_{1}}\right\} \cap\left\{y_{i_{2}}, y_{j_{2}}\right\}=\emptyset$, where $\pi_{1}^{-1}(k)=\left\{i_{1}, j_{1}\right\}$ and $\pi_{2}^{-1}(k)=\left\{i_{2}, j_{2}\right\}$. Equivalently, we set an edge if $T\left(x_{\pi_{1}^{-1}(k)}, y_{\pi_{2}^{-1}(k)}\right) \neq 0$ for all $k \in\{1, \ldots, R\}$,

This completes the construction of $G^{\prime}$.

Completeness: Assume there is a labeling $L$ that satisfies all constraints of $\Phi$. Define the coloring of $G^{\prime}$ to be $c(w, x)=x_{L(w)}$ for all $w \in W$ and $x \in\{0,1,2,3\}^{2 R}$. We show that this is a legal coloring of $G^{\prime}$. Indeed, let $\left(\left(w_{1}, x\right),\left(w_{2}, y\right)\right) \in E^{\prime}$ be an edge of $G^{\prime}$. Then $w_{1}, w_{2}$ have a common neighbor $v \in V$. Let $\pi_{1}$ and $\pi_{2}$ be the corresponding constraints, and let $k=L(v)$. Then $\pi_{1}\left(L\left(w_{1}\right)\right)=k=\pi_{2}\left(L\left(w_{2}\right)\right)$, as $L$ satisfies all the constraints.

Since $\left(\left(w_{1}, x\right),\left(w_{2}, y\right)\right) \in E^{\prime}$ the sets $x_{\pi_{1}^{-1}(k)}$ and $y_{\pi_{2}^{-1}(k)}$ are disjoint, and hence $c\left(w_{1}, x\right) \neq$ $c\left(w_{2}, y\right)$ as $c\left(w_{1}, x\right)=x_{L\left(w_{1}\right)} \in x_{\pi_{1}^{-1}(k)}$ and $c\left(w_{2}, y\right)=y_{L\left(w_{2}\right)} \in y_{\pi_{2}^{-1}(k)}$.

Soundness: Suppose that $G^{\prime}$ contains an independent set $S \subseteq V^{\prime}$ such that $\frac{|S|}{\left|V^{\prime}\right|} \geq \epsilon$. Our goal is to show that is such case $\operatorname{val}(\Phi)>\operatorname{poly}(\epsilon)$. Let $J$ be a subset of $W$ that make a non-negligible contribution to $S$

$$
J=\left\{w \in W: \frac{[w] \cap S}{[w]}>\frac{\epsilon}{2}\right\} .
$$

By Markov inequality we have $|J| \geq \frac{\epsilon}{2}|W|$.

For each $w \in J$ let $f_{w}:\{0,1,2,3\}^{2 R} \rightarrow\{0,1\}$ be the indicator function of $S$, i.e., $f_{w}(x)=1$ if and only if $(w, x) \in S$. Then $\mathbb{E}\left[f_{w}\right]>\epsilon / 2$ for each such $w \in J$. Let $\delta=\epsilon^{O(1)}$ and $k=O(\log (1 / \epsilon))$ as assured by Theorem 1.3 when it is applied on the operator $T$ from Definition 4.2 with parameter $\epsilon / 2$. Next for each $w \in J$ we define a small set of labels

$$
L(w)=\left\{i: \operatorname{Inf}_{i}^{\leq 2 k}\left(f_{w}\right)>\delta / 2\right\} .
$$

By Proposition 2.4 we have $|L(w)|<\frac{4 k}{\delta}$. Next we give labels to neighbors of $J$ in $\Phi$. 
Claim 4.3 Let $v \in N(J)$. Let $w_{1}, w_{2} \in N(v) \cap J$, and let $\pi_{1}, \pi_{2}$ be the corresponding constraints. Then, there are $i \in L_{w_{1}}$ and $j \in L_{w_{2}}$ such that $\pi_{1}(i)=\pi_{2}(j)$.

Proof Recall that $f_{w}$ 's are indicators of an independent set. Thus $f_{w_{1}}(x)=1=f_{w_{2}}(y)$ implies that $\left(\left(w_{1}, x\right),\left(w_{2}, y\right)\right) \notin E^{\prime}$. Therefore, $T\left(x_{\pi_{1}^{-1}(k)}, y_{\pi_{2}^{-1}(k)}\right)=0$ for some $k \in$ $\{1, \ldots, R\}$, and thus

$$
T^{\otimes R}\left(\left(x_{\pi_{1}^{-1}(1)}, \ldots, x_{\pi_{1}^{-1}(R)}\right),\left(y_{\pi_{2}^{-1}(1)}, \ldots, y_{\pi_{2}^{-1}(R)}\right)\right)=0 .
$$

Define

$$
\bar{f}\left(x_{\pi_{1}^{-1}(1)}, \ldots, x_{\pi_{1}^{-1}(R)}\right)=f_{w_{1}}\left(x_{1}, \ldots, x_{2 R}\right)
$$

and

$$
\bar{g}\left(y_{\pi_{2}^{-1}(1)}, \ldots, y_{\pi_{2}^{-1}(R)}\right)=f_{w_{2}}\left(y_{1}, \ldots, y_{2 R}\right),
$$

where we think of $\bar{f}, \bar{g}$ as functions in $R$ variables, each taking values in $\{0,1,2,3\}^{2}$. We show that $\left\langle\bar{f}, T^{\otimes R} \bar{g}\right\rangle=0$. Then, using Theorem 1.3, we conclude that there is $\ell \in\{1, \ldots, R\}$ such that $\operatorname{Inf}_{\bar{\ell}}^{\leq k}(\bar{f})>\delta$ and $\operatorname{Inf}_{\bar{\ell}}^{\leq k}(\bar{g})>\delta$. Using the relation between $\bar{f}$ and $f_{w_{1}}$ we conclude that there is some $i \in \pi_{1}^{-1}(\ell)$ such that $\operatorname{Inf}_{i}^{\leq 2 k}\left(f_{w_{1}}\right)>\delta / 2$. Similarly for $g$ there is some $j \in \pi_{2}^{-1}(\ell)$ such that $\operatorname{Inf}_{j}^{\leq 2 k}\left(f_{w_{2}}\right)>\delta / 2$. Therefore, there are $i \in L_{w_{1}}, j \in L_{w_{2}}$ such that $\pi_{1}(i)=\pi_{2}(j)$.

It is left to show that $\left\langle\bar{f}, T^{\otimes R} \bar{g}\right\rangle=0$. Indeed,

$$
\begin{aligned}
\left\langle\bar{f}, T^{\otimes R} \bar{g}\right\rangle & =\frac{1}{4^{2 R}} \sum_{x \in\left(\{0,1,2,3\}^{2}\right)^{R}} \bar{f}(x) T^{\otimes R} \bar{g}(x) \\
& =\frac{1}{4^{2 R}} \sum_{x} \bar{f}(x) \sum_{y} T^{\otimes R}(x, y) \bar{g}(y) \\
& =\frac{1}{4^{2 R}} \sum_{\substack{x: f_{w_{1}}(x)=1 \\
y: f_{w_{2}}(y)=1}} T^{\otimes R}\left(x_{\left.\pi_{1}^{-1}, y_{\pi_{2}^{-1}}\right)}\right. \\
& =\frac{1}{4^{2 R}} \sum_{\substack{x: f_{w_{1}}(x)=1 \\
y: f_{w_{2}}(y)=1}} 0 \\
& =0, \quad
\end{aligned}
$$

as required.

From the claim above we get that for all $v \in N(J)$ and any $w_{1}, w_{2} \in N(v) \cap J$ it holds that

$$
\operatorname{Pr}_{\substack{i \in L\left(w_{1}\right) \\ j \in L\left(w_{2}\right)}}\left[\pi_{1}(i)=\pi_{2}(j)\right] \geq \frac{1}{\left|L\left(w_{1}\right)\right|\left|L\left(w_{2}\right)\right|} \geq\left(\frac{\delta}{4 k}\right)^{2} .
$$

By averaging there is $L_{0}: V \cup W \rightarrow\{1, \ldots, 2 R\}$ such that for all $w \in J$ we have

$$
\operatorname{Pr}_{v \in N(w)}\left[L_{0}(v)=\pi\left(L_{0}(w)\right)\right] \geq\left(\frac{\delta}{4 k}\right)^{2} .
$$


Hence, if we assume regularity on the vertices of $W$, we get

$$
\begin{aligned}
\operatorname{Pr}_{(v, w) \in E}\left[L_{0}(v)=\pi\left(L_{0}(w)\right)\right] & \geq \operatorname{Pr}_{w \in W}[w \in J] \operatorname{Pr}_{v \in N(w)}\left[L_{0}(v)=\pi\left(L_{0}(w)\right) \mid w \in J\right] \\
& \geq \epsilon\left(\frac{\delta}{4 k}\right)^{2} \\
& =\operatorname{poly}(\epsilon) .
\end{aligned}
$$

We conclude that $\operatorname{val}(\Phi)>$ poly $(\epsilon)$, which completes the soundness analysis of the reduction. Theorem 1.2 follows.

\section{Acknowledgments}

We are thankful to Oded Regev for useful comments on the paper.

\section{References}

[AC06] S. Arora and E. Chlamtac. New approximation guarantee for chromatic number. In STOC '06: Proceedings of the thirty-eighth annual ACM Symposium on Theory of Computing, pages 215-224, 2006.

$\left[\mathrm{ALM}^{+} 98\right]$ S. Arora, C. Lund, R. Motwani, M. Sudan, and M. Szegedy. Proof verification and hardness of approximation problems. Journal of the ACM, 45(3):501-555, 1998.

[AS98] S. Arora and S. Safra. Probabilistic checking of proofs : a new characterization of NP. Journal of the ACM, 45(1):70-122, 1998.

[BK97] A. Blum and D. Karger. An $\widetilde{O}\left(n^{3 / 14}\right)$-coloring algorithm for 3-colorable graphs. Information Processing Letters, 61:49-53, 1997.

[BOL89] M. Ben-Or and N. Linial. Collective coin flipping. Randomness and Computation, pages $91-115,1989$.

[Chl07] E. Chlamtac. Approximation algorithms using hierarchies of semidefinite programming relaxations. In FOCS '0\%: Proceedings of the 48th Annual IEEE Symposium on Foundations of Computer Science, pages 691-701, 2007.

[DMR09] I. Dinur, E. Mossel, and O. Regev. Conditional hardness for approximate coloring. SIAM Journal on Computing, 39(3):843-873, 2009.

[GK04] V. Guruswami and S. Khanna. On the hardness of 4-coloring a 3-colorable graph. SIAM J. Discret. Math., 2004.

[Kar72] R. Karp. Reducibility among combinatorial problems. Complexity of Computer Computations, 43:85-103, 1972.

[Kho02] S. Khot. On the power of unique 2-prover 1-round games. In Proceedings of the 34th Annual ACM Symposium on Theory of Computing, pages 767-775, 2002. 
[KLS00] S. Khanna, N. Linial, and S. Safra. On the hardness of approximating the chromatic number. Combinatorica, 20(3):393-415, 2000.

[KMS98] D. Karger, R. Motwani, and M. Sudan. Approximate graph coloring by semidefinite programming. J. ACM, 45(2):246-265, 1998.

[KPW04] E. De Klerk, D.V. Pasechnik, and J.P. Warners. On approximate graph colouring and max-k-cut algorithms based on the $\vartheta$-function. Journal of Combinatorial Optimization, 8(3):267-294, 2004.

[KT12] K. Kawarabayashi and M. Thorup. Combinatorial coloring of 3-colorable graphs. FOCS '12: Proceedings of the 53rd Annual IEEE Symposium on Foundations of Computer Science, pages 68-75, 2012.

[MOO10] E. Mossel, R. O'Donnell, and K. Oleszkiewicz. Noise stability of functions with low influences: Invariance and optimality. Annals of Mathematics, 171(1):295341,2010 .

[RR01] Y. Rinott and V. Rotar'. A remark on quadrant normal probabilities in high dimensions. Statistics and Probability Letters, 51(1):47-51, 2001. 